\title{
Schnitzler's syndrome: A female elderly case presenting intractable non-pruritic febrile urticarial rush
}

\author{
Nanae Kimura, ${ }^{1,2}$ Hiromichi Takeshita, ${ }^{2}$ Tatsuya Kai, ${ }^{3}$ Yasushi Inoue, ${ }^{3}$ Masutaka Furue ${ }^{1}$
}

\begin{abstract}
Schnitzler's syndrome is an acquired autoinflammatory disease characterized by chronic urticarial rash and monoclonal gammopathy (predominantly IgM type). A 75-year-old Japanese woman complained of high fever and non-pruritic urticarial rash appearing almost every day for 3 years. Her abnormal laboratory data included leukocytosis and neutrophilia with elevated erythrocyte sedimentation rate and C-reactive protein level. Hyperglobulinemia of IgA and IgM was also noted. Histological analysis revealed perivascular and interstitial neutrophilic infiltration without any signs of vasculitis. Immunofixation analysis confirmed IgM-kappa-type monoclonal gammopathy. Oral prednisolone initially improved her symptoms, but recurrence was observed upon its tapering. The addition of colchicine successfully controlled her symptoms and allowed a reduction in the dose of systemic steroid.
\end{abstract}

Key words: Schnitzler's syndrome, IL-1 $\beta$, acquired autoinflammatory disease, urticarial rash, monoclonal gammopathy

\section{From:}

${ }^{1}$ Graduate School of Medical Sciences, Department of Dermatology, Kyushu University, Fukuoka, Japan

${ }^{2}$ Dermatology Unit, Japanese Red Cross Fukuoka Hospital, Fukuoka, Japan

${ }^{3}$ Collagen Diseases Unit, Japanese Red Cross Fukuoka Hospital, Fukuoka, Japan

\section{Introduction}

Schnitzler's syndrome is an acquired autoinflammatory disease characterized by chronic "non-pruritic" urticarial rash and monoclonal gammopathy (predominantly IgM type), which was first reported by the French dermatologist Liliane Schnitzler in $1972 .{ }^{1}$ Recently, much attention has focused on the role of interleukin-1 beta (IL-1 $\beta$ ) as a pathogenetic trigger in this disease. ${ }^{2,3}$

\section{Report of Case}

A 75-year-old Japanese woman complained of high fever and urticarial rash appearing almost every day for 3 years. Examination revealed an intermittent fever up to $39^{\circ} \mathrm{C}$ and disseminated non-pruritic urticarial rash, which had lasted for several days (Figure 1a), with fatigue, sweating, and sacroiliac joint pain. Treatments for diabetes had been undergoing for several years.

Laboratory evaluation showed leukocytosis $\left(22.5 \times 10^{3} / \mu \mathrm{L}\right)$ and neutrophilia $\left(18.3 \times 10^{3} / \mu \mathrm{L}\right)$ with elevated erythrocyte sedimentation rate of $70 \mathrm{~mm} / \mathrm{h}$ and C-reactive protein (CRP) level of $21.22 \mathrm{mg} / \mathrm{dl}$. Slight elevation of ferritin (334.9; normal range, 3.6 to $114.0 \mathrm{ng} / \mathrm{ml}$ ) was also observed. In addition, hyperglobulinemia of $\operatorname{IgA}(525 \mathrm{mg} / \mathrm{dl})$ and $\operatorname{IgM}(253 \mathrm{mg} / \mathrm{dl})$ was noted.

\author{
Corresponding author: \\ Nanae Kimura \\ Graduate School of Medical Sciences, Department of Dermatology, \\ Kyushu University, \\ 3-1-1, Maidashi, Higashi-ku, Fukuoka city, Fukuoka, 812-8582, Japan \\ E-mail: nanae@dermatol.med.kyushu-u.ac.jp
}

Antinuclear antibody, anti-neutrophil cytoplasmic antibodies, and anti-human T-cell leukemia virus antibody were all negative. CT scan revealed only reactive axillary and inguinal lymphadenopathy. Bone marrow aspiration showed no hematopoietic malignancies.

Histological analysis revealed perivascular and interstitial neutrophilic infiltration without any signs of vasculitis. Dermal edema was very mild (Figure 1b). These features are classified as neutrophilic urticarial dermatosis and urticarial vasculitis was denied. ${ }^{2}$

Regarding hyperglobulinemia, we further performed serum electrophoresis and immunofixation. While serum electrophoresis indicated no particular abnormality, we identified abnormal bands for both IgM and kappa chain by immunofixation (Figure 1c). Furthermore, an abnormal band for kappa chain was also detected by urine immunofixation (data not shown), indicating a diagnosis of Schnitzler's syndrome with IgM-kappa-type monoclonal gammopathy. ${ }^{1}$

Systemic prednisolone at an initial dose of $30 \mathrm{mg} /$ day successfully improved the patient's fever and skin rash with normalization of leukocytosis and CRP level. Tapering the dose of prednisolone led to recurrence of her skin rash and additional 

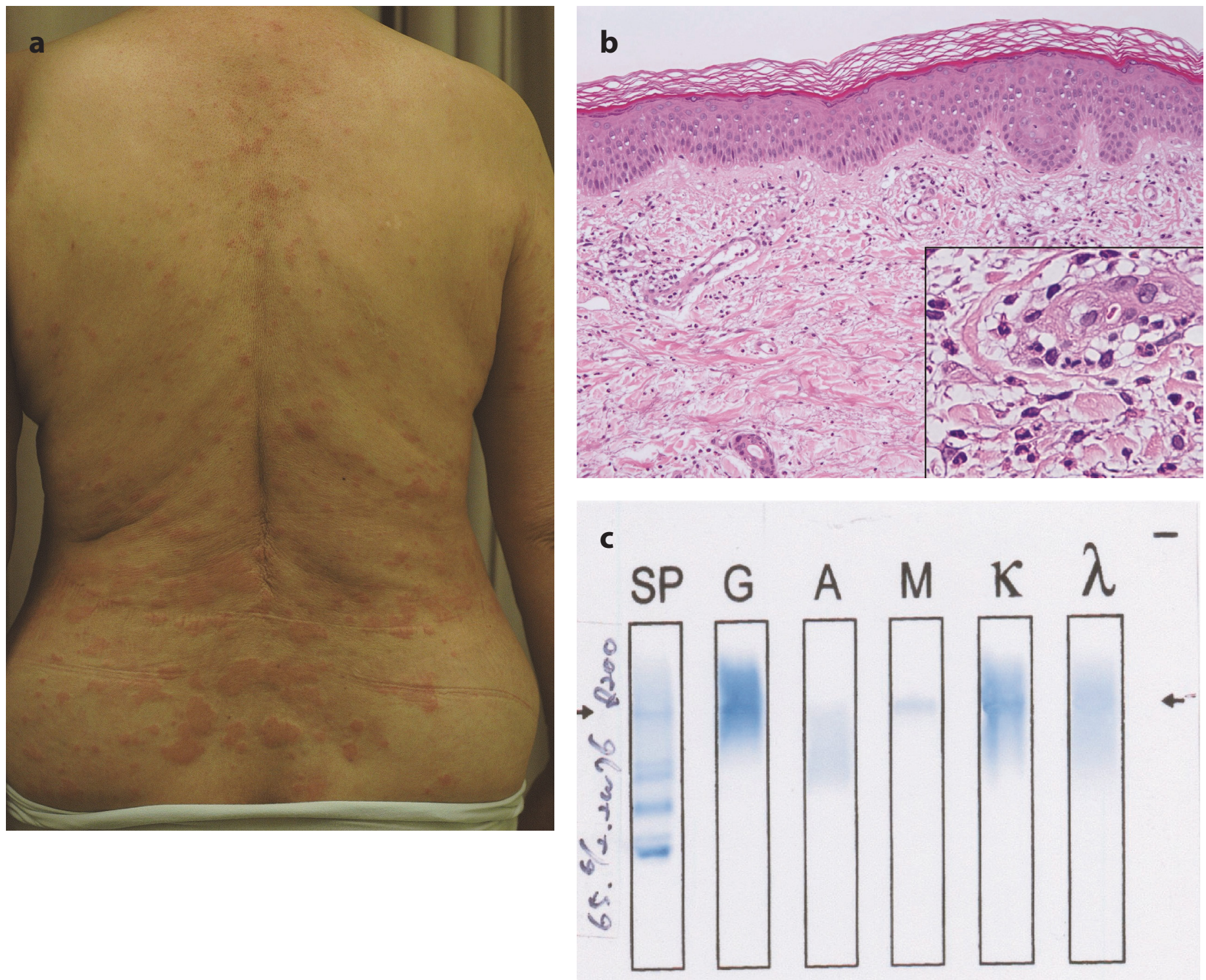

Figure 1.

a. Disseminated urticarial eruptions on the trunk. They were non-pruritic and persisted for several days.

b. Perivascular and interstitial neutrophilic infiltration was seen without any vasculitis. Dermal edema was mild.

c. Abnormal bands for both IgM and kappa chain were identified by immunofixation.

intake of azathioprine at $50 \mathrm{mg} /$ day did not help to inhibit the leukocytosis and elevation of CRP. After the cessation of azathioprine, oral colchicine was initiated at $0.5 \mathrm{mg} / \mathrm{day}$, which led to dramatic subsidence of the symptoms and improvement of the laboratory findings. Good control of the patient's symptoms was achieved with $12.5 \mathrm{mg} /$ day prednisolone. Serum IgM levels kept high, $253 \mathrm{mg} / \mathrm{dl}$ to $561 \mathrm{mg} / \mathrm{dl}$, during 5-month follow up period.

\section{Discussion}

For making the correct diagnosis of Schnitzler's syndrome, differential diagnoses were considered and excluded following Strasbourg diagnostic criteria. ${ }^{1}$ Adult-onset Still's disease (AOSD) has similar clinical characteristics such as intermittent fever, pruritic urticarial rash and leukocytosis. We ruled out AOSD due to lack of significant increase of transaminases and ferritin. But her urticaria was "non-pruritic" and she presented
IgM gammopathy. Other autoinflammatory diseases such as familial cold autoinflammatory syndrome, Muckle-Wells syndrome, neonatal-onset multisystem inflammatory disease and familial Mediterranean fever were also excluded by negative familial or infantile history and neurological or ophthalmological symptoms. Urticarial vasculitis was denied by histological analysis. We also ruled out Sweet syndrome based on clinical and histological manifestation of her eruption. ${ }^{3}$

Since it was proposed that IL-1 signaling plays a critical role in Schnitzler's syndrome, the anti-IL-1 antibodies anakinra and canakinumab are currently recommended as first-line treatment. ${ }^{1}$ However, they are still off-label treatments in Japan. Systemic steroids are instead the mainstay in Japan, and colchicine may work as a second-line medicine, as demonstrated in the present case. Simons et al. recommended colchicine as one of the main treatments for none-severe cases without significant alterations in quality of life or persistent elevation of 
inflammation markers. ${ }^{1}$ Their survey revealed that colchicine seemed to be beneficial in $25 \%$ of treated patients. Colchicine is also recommended as an additional treatment for anakinra-non-responders. ${ }^{1}$

De Koning reported that the median age of fever onset was 52 years old in 66 patients with Schnitzler's syndrome. ${ }^{2}$ Our patient's onset was in her seventies which was relatively older than reported cases. To avoid worsening of comorbid diabetes and additional risk of osteoporosis in the elderly patient, we attempted to taper the prednisolone dose but failed. However, addition of colchicine was successful in controlling the symptoms and reducing the systemic steroid dose.

When evaluating patients with fever of unknown origin, systemic examination is mandatory to detect infectious focus, collagen diseases and malignant diseases. Dermatological features, if present, are also important clues for a proper diagnosis. Chronic urticaria and AOSD basically manifest "pruritic" wheal. In contrast, "non-pruritic" wheal is the characteristic feature of Schnitzler's syndrome. In addition, histological analysis of biopsied skin would help rapid and collect differential diagnosis.
Another important issue in this acquired autoinflammatory disease is possible progression to malignant lymphoproliferative disorders. ${ }^{1,2,4}$ As increased levels of serum IgM are probably long-lasting as shown in the present case, careful hematologic monitoring is thus mandatory in Schnitzler's syndrome.

\section{References}

1. Simon A, Asli B, Braun-Falco M, de Koning HD, Fermand J-P, Grattan C, et al. Schnitzler's syndrome: diagnosis, treatment, and follow-up. Allergy. 2013;68:562-8

2. de Koning HD. Schnitzler's syndrome: lessons from 281 cases. Clin Transl Allergy. 2014;4:41.

3. Su WP, Liu HN. Diagnostic criteria for Sweet's syndrome. Cutis. 1986; 37:167-74.

4. Gusdorf L, Lipsker D. Schnitzler's syndrome: a review. Curr Rheumatol Rep. 2017;19:46. 